\title{
Cosmology from quantum information
}

\author{
César Gómez \\ Instituto de Física Teórica UAM-CSIC, Universidad Autónoma de Madrid, \\ Cantoblanco, 28049 Madrid, Spain \\ Raul Jimenez \\ ICC, University of Barcelona, Marti i Franques 1, 08028 Barcelona, Spain \\ and ICREA, Pg. Lluis Companys 23, Barcelona E-08010, Spain
}

(Received 19 February 2020; accepted 27 August 2020; published 11 September 2020)

\begin{abstract}
We describe inflation in terms of a time-dependent quantum density matrix with time playing the role of a stochastic variable. Using a quasi-de Sitter model we compute the corresponding quantum Fisher information function as the second derivative of the relative entanglement entropy for the density matrix at two different times. Employing standard quantum estimation theory we evaluate the minimal variance of quantum scalar fluctuations that reproduces the power spectrum and the corresponding tilt in the slow-roll limit. The Jeffreys prior associated with such Fisher information can be used to define the probabilities on the set of initial conditions defined by the slow-roll parameter $\epsilon$ and the initial Shannon information.
\end{abstract}

DOI: 10.1103/PhysRevD.102.063511

The current cosmological model is the $\Lambda \mathrm{CDM}$, whose seven free parameters are well constrained, at the percent level, by the data from the Planck mission [1]. This model provides a remarkable description of a wealth of cosmological observations. This model, in its early phase, postulates an epoch of accelerated expansion that leads to a very large and homogeneous Universe. After this supercooled expansion phase, reheating takes place; in this phase of reheating the inflaton converts its energy into particles via resonant coupling to the standard model of particle physics. This hot big bang phase further leads to a matter dominated Universe. This early inflationary epoch is crucial to explain the flatness and the present homogeneity of the observed Universe [2-4]. The quantum origin of the large scale structure is explained on the basis of the classicalization of small quantum fluctuations that leave the horizon during the inflationary epoch and reenter during the hot big bang period [5-8]. This is achieved if the time evolution of the horizon changes from shrinking during the inflationary epoch to increasing after reheating. While we have not yet measured the smoking gun of an exponential phase of expansion in the early Universe (the much expected primordial B-mode polarization of the cosmic microwave background), there are two remarkable measurements that strongly support inflation as the correct

Published by the American Physical Society under the terms of the Creative Commons Attribution 4.0 International license. Further distribution of this work must maintain attribution to the author(s) and the published article's title, journal citation, and DOI. Funded by SCOAP . picture to describe the early Universe: the existence of superhorizon fluctuations [9] and the fact that the power spectrum of matter fluctuations is nearly scale invariant and has a red tilt [1].

There is however not a definite picture of the physics behind inflation. The usual approach is to postulate a potential from a hypothetical scalar field and adjust this potential in order to fit the observational constraints. The recent Planck [1] analysis already constrains this potential to be very flat. In this paper we try a different approach to describe the epoch of inflation and the early Universe.

Our guiding principle will be quantum information and more precisely quantum estimation theory (see Ref. [10]). ${ }^{1}$ From the point of view of a quantum mechanical description, we are interested in the time dependence of a set of cosmological observables. Since time itself in quantum mechanics is not an observable, we interpret it as a stochastic variable and we encode the information about time dependence in terms of a Fisher function. Through its relation with the relative entanglement entropy (for a review see e.g., [14]) that defines a natural distance between the quantum states of the early Universe at different times, we introduce a simple quasi-de Sitter model to estimate the quantum Fisher function.

Using the standard relations between the Fisher function and the variance of the associated stochastic quantity, we reproduce some of the most basic results about inflation, in particular, bounds on the duration of inflation as well as the

\footnotetext{
${ }^{1}$ For recent applications of quantum estimation theory in the same spirit see Refs. [11-13].
} 
power spectrum of scalar quantum fluctuations. This is done without assuming any particular form for the inflaton potential. An interesting and potentially deep output of this approach is the important role of the saturation of minimal variance on the basis of the quantum Cramer-Rao inequality that sets the variance of cosmological fluctuations as given by the power spectrum at the point of horizon exit.

As a first approximation we can try to describe the inflationary epoch in terms of a time-dependent Hubble parameter $H$ that is postulated to decrease i.e., $\dot{H} \leq 0$. Quantum mechanically we can describe the state of the Universe at time $t$ using a quantum density matrix $\rho(t)$ that we can define by taking the trace of the quantum state over the whole region outside the horizon at time $t .^{2}$ Now we can define the relative entanglement entropy between two different times as

$$
S\left(t ; t_{0}\right) \equiv \operatorname{Tr}\left(\rho(t) \ln \frac{\rho(t)}{\rho\left(t_{0}\right)}\right)
$$

for $t_{0}$ the initial time. As it is well known in information theory, $S\left(t ; t_{0}\right)$ is positive definite and leads to the first law of entanglement, namely

$$
\dot{S}(t)=0
$$

If we Taylor expand around $t_{0}$ we get

$$
S\left(t_{0}+\delta_{t} ; t_{0}\right)=S(0)+\frac{1}{2} \delta_{t}^{2} F\left(t_{0}\right)
$$

with $F\left(t_{0}\right)$ the quantum Fisher information at the initial time $t_{0}$. Note from the definition (1) that $S(0)=0$. We shall characterize the initial condition of our cosmology in terms of the value of the initial Fisher information. In statistical terms, the time $t$ is working like a stochastic variable i.e., time is just defining the variable space and the Fisher function defines the metric on this space.

The relative entropy can be represented as

$$
S\left(t ; t_{0}\right)=\operatorname{Tr}(\rho(t) \mathcal{H})-\operatorname{Tr}\left(\rho\left(t_{0}\right) \mathcal{H}\right)-S(\rho(t))+S\left(\rho\left(t_{0}\right)\right)
$$

for $S(\rho)$ the standard von Neumann entropy and $\mathcal{H}$ the modular or entanglement Hamiltonian. If the density matrix is Hermitian and positive semidefinite it can be always expressed as $\rho=\frac{e^{-\mathcal{H}}}{\operatorname{tr}\left(e^{-\mathcal{H}}\right)}$ for $\mathcal{H}$ some Hermitian operator. This is the operator defining the modular Hamiltonian. In essence the modular Hamiltonian represents the density matrix as a formal canonical matrix. In general, to find the modular Hamiltonian for a generic density matrix is a very

\footnotetext{
${ }^{2}$ Assuming the quantum state of the Universe at a given time is $|\Psi\rangle$ we define this density matrix formally as $\operatorname{Tr}|\Psi\rangle\langle\Psi|$ with the trace over the region outside the horizon at that time. In what follows we will not need a concrete characterization of the state $|\Psi\rangle$.
}

difficult task. In our case we will profit from representing the quasi-de Sitter density matrix as thermal for the Gibbons-Hawking temperature [15]. Once we assume $t$ close to $t_{0}$ and we choose for $\rho\left(t_{0}\right)$ a canonical density matrix at temperature $T$, the relative entropy can be represented as

$$
S\left(t ; t_{0}\right)=\frac{1}{T}\left(\mathcal{F}(\rho(t))-\mathcal{F}\left(\rho\left(t_{0}\right)\right)\right)
$$

with $\mathcal{F}(\rho)$ the free energy i.e., $(E-s T)$ for $E$ the energy and $s$ the entropy. We will use this representation in order to estimate the relative entropy. Moreover the quantum Fisher function can be written as

$$
F(t)=\operatorname{Tr}\left(\rho(t) L_{t}^{2}\right)
$$

for $L_{t}$ the Lyapunov operator driving the time evolution of $\rho .^{3}$ Models of inflation are in fact ways to model $L_{t}$ and consequently also $\mathcal{H}$. In what follows we will use to estimate the Fisher function a simple model where we only assume that $H$ depends on time and that $\dot{H}$ is negative.

Before going into the detailed computation, let us highlight the key point of our approach in more qualitative terms. In general relativity, canonical quantizations lead to the Wheeler-de Witt equation which does not involve time. To recover standard time evolution it requires one to introduce a clock field, let us say $\phi .{ }^{4}$ In cosmology this clock field is just the inflaton. Once we have defined, using this clock, time evolution, we can formally compute the associated Lyapunov operator that depends on the corresponding clock. This allows us to compute the associated quantum Fisher function $F_{\phi}$ using (6). Our main claim is that the corresponding power spectrum $\Delta_{\phi}^{2}$ is just determined by the inverse of the quantum Fisher function

$$
\Delta_{\delta \phi}^{2} \sim \frac{1}{F_{\phi}} .
$$

In other words, once we introduce a clock to paramtrize the time dependence, the corresponding quantum variance defines the power spectrum of fluctuations of the field used to define the clock $^{5}$

In what follows we will estimate the cosmological quantum Fisher function using its relation with the relative entropy (3). We shall define $S\left(t ; t_{0}\right)$ using $(5)$ as $(E(t) / T-$ $s(t))-\left(E\left(t_{0}\right) / T-s\left(t_{0}\right)\right)$ for $s(t), E(t), T$ respectively entropy, energy, and effective temperature. Moreover we

\footnotetext{
${ }^{3}$ This operator simply represents the symmetric logarithmic derivative and it is defined by the Lyapunov equation $\frac{d \rho}{d t}=\frac{L_{t} \rho+\rho L_{t}}{2}$.

${ }_{5}^{4}$ See for instance the discussion in Ref. [16].

${ }^{5} \mathrm{As}$ an aside remark let us just say that the physical nature of the clock field by contrast to just a Stuelkeberg field making formally the theory invariant under reparametrizations lies in the finiteness of the corresponding quantum Fisher function.
} 
will take the same $T$ as defined by the canonical density matrix at time $t^{6}$

$$
\rho(t)=\frac{e^{E(t) / T}}{\operatorname{Tr}\left(e^{E(t) / T}\right)}
$$

For a given time characterized by $H(t)$ we define, in Planck units,

$$
S\left(t ; t_{0}\right)=\frac{(a-b)}{H^{2}}-\frac{a}{H H_{0}}+\frac{b}{H_{0}^{2}}
$$

for $a, b$ two parameters with units of $M_{P}^{2}$ and with $H_{0}$ the Hubble rate at the initial time. The logic of this model of the relative entropy is based on assuming the following quaside Sitter relations: $T \sim H, E(t) \sim \frac{H(t)^{2}}{H(t)^{3}}$ and $s(t) \sim \frac{1}{H(t)^{2}}$, all of them in natural Planck units. The temperature $T$ is simply defined as the Gibbons-Hawking temperature [15] for a de Sitter with Hubble parameter $H$. Infinitesimally around a reference point we define the difference in free energy keeping the same $T$ and changing the entropy and the energy accordingly with the de Sitter relations. This approximation leads to (9) as a phenomenological model of the relative entropy.

Note that (9) satisfies $S\left(t_{0} ; t_{0}\right)=0$ and that this model of relative entropy is well defined at the inflationary epoch since $S\left(t ; t_{0}\right)$ is always positive for any time $t>t_{0}$. Secondly, in order to satisfy the first law of entanglement, namely $\dot{S}=0$, in (9), $a=2 b$. Now we can easily compute the Fisher function:

$$
F\left(t_{0}\right)=2 b \epsilon^{2}
$$

where we have introduced the slow-roll parameter

$$
\epsilon=-\frac{\dot{H}}{H^{2}}
$$

We have only used the assumption that $H$ changes with time and we define $\epsilon$ in a way that is not assuming any underlying inflaton potential model. Thus we observe that the slow roll simply generates a nonvanishing Fisher function. Using now the Cramer-Rao inequality, this finite Fisher function can be used to define the uncertainty on time, which is bounded by the minimal uncertainty

$$
\Delta_{\delta t} \sim \frac{1}{\sqrt{2 b} \epsilon} .
$$

As it is well known, this relation is the statistical version of the quantum energy time uncertainty principle. Saturating

\footnotetext{
${ }^{6}$ Physically this means that locally we assume that the system tends to equilibrium with time. More precisely we assume that the thermal equilibrium matrix is the one at $t$ for $t$ in the past of $t_{0}$.
}

the Cramer-Rao inequality is equivalent to minimizing the corresponding uncertainty.

What we can call the minimal statistical duration of inflation is determined by the initial value $\epsilon$ by (12). This is measured in Planck units. To change to Hubble units, and taking $2 b$ order one, we get

$$
\Delta_{\delta t}=\frac{\mathcal{N}}{\sqrt{N_{0}}}
$$

Hubble times. Here $\mathcal{N}$ is the number of e-foldings and $N_{0} \equiv \frac{M_{P}^{2}}{H_{0}^{2}}$. So if we tune the initial Hubble to be Planckian, the saturation of Cramer-Rao sets the duration of inflation. In other words the physical duration of inflation, given in Hubble units as the number of e-foldings, let us say $\Delta_{\text {phys }}$, is given by

$$
\Delta_{\text {phys }} \sim \sqrt{N_{0}} \Delta_{\delta t} .
$$

In summary, from the information point of view, the minimal step in the cosmological evolution ${ }^{7}$ is set by the initial Fisher information and is given by $\Delta_{\delta t}$. The number of cosmological steps that we can identify as the complexity of the whole inflation process is determined by $\sqrt{N_{0}}$ and therefore is established by the initial Shannon information.

Note that the information description of the inflationary phase makes sense only if $\epsilon<1$. This means that to extend inflation beyond $\epsilon=1$ makes no sense in information theory unless one assumes sub-Planckian resolution of time. In other words, whenever the Universe reaches $\epsilon=1$, information theory is already implying that the inflationary period cannot continue, very much as in the standard case. In this sense we fully reproduce the standard criteria for the end of inflation, namely $\epsilon>1$, but we read the necessity of this condition from information theory as the impossibility of time resolutions smaller than Planck time. In other words we simply provide a very simple argument why the system cannot stay in the inflationary phase once $\epsilon$ becomes bigger than one.

The information content of the initial state of the Universe is, therefore, a Shannon information $N_{0}$ and a Fisher information. If we assume an inflationary epoch with decreasing Hubble parameter, the Fisher information sets the corresponding rate of change. The limiting case $\epsilon=0$ leads to vanishing Fisher information. Using the qualitative relation between Fisher and Shannon for the Gaussian case [17], and assuming it can be generalized, will imply that $\epsilon=0$ is only consistent with $N_{0}=\infty$.

Let us now briefly discuss the crucial issue of generation of seeds for galaxy formation as quantum fluctuations. Until now we have discussed the Fisher function associated

\footnotetext{
${ }^{7}$ In computational language the time of the elementary "cosmological gate."
} 
with time as a stochastic variable and discover that $F$ goes like $\epsilon^{2}$. This means that the corresponding variance goes like

$$
\Delta_{\delta t}^{2} \sim \frac{1}{\epsilon^{2}} .
$$

Physically one can figure out the meaning of this expression as defining the variance of time on a surface of constant energy density.

This effective variance in time induces in general relativity the curvature fluctuations. In essence, the quantum fluctuations in inflation have as origin that inflation ends at different times in different regions of space. To describe this phenomena we simply use the variance of time (interpreted as a stochastic variable) induced by the initial Fisher function.

If we are interested in the expression of these fluctuations for a hypothetical inflaton field $\phi$ we can use the relation $\delta \phi=\dot{\phi} \delta t$. The quantity that is now dependent on the particular model of inflation is $\dot{\phi}$. In the slow-roll approximation and in Planck units we can use

$$
\dot{\phi}^{2} \sim H^{2} \epsilon
$$

This leads to the variance of $\delta \phi$ corresponding to the power spectrum

$$
\Delta_{\delta \phi}^{2} \sim \dot{\phi}^{2} \Delta_{\delta t}^{2} \sim \frac{H^{2}}{\epsilon}
$$

which is the expected result for the power spectrum of scalar perturbations [5]. In other words, the Fisher function for $\phi$, i.e., when we take the inflaton scalar field as a stochastic variable, controls the power spectrum of curvature perturbations through the Cramer-Rao bound

$$
\Delta_{\delta \phi}^{2} \sim \frac{1}{F_{\phi}}
$$

with $F_{\phi}=\frac{\epsilon}{H^{2}}$.

At this point it would be worth commenting on the relation between this statistical derivation and the standard derivation of the power spectrum for the curvature perturbations. In this second case the time fluctuations at the end of inflation, on a field hypersurface, let us say $\delta t$, induce curvature perturbations of order $H \delta t$ and therefore a power spectrum that goes like $H^{2}(\delta t)^{2}$ with $\delta t \sim \frac{1}{\sqrt{\epsilon}}$. We are instead representing the curvature power spectrum as $\dot{\phi}^{2}(\Delta t)^{2}$ with $\Delta t$ defined by the Fisher function as the total duration of the process of inflation i.e., $\frac{1}{\epsilon}$. Thus the power spectrum of curvature perturbations is simply defined as $(\Delta \phi)^{2}=\dot{\phi}^{2}(\Delta t)^{2}$ or equivalently $(\Delta \phi)^{2} \sim$ $\langle\delta \phi \delta \phi\rangle \Delta(t)$ with the standard value of $\langle\delta \phi \delta \phi\rangle \sim H^{2}$.

It is interesting to observe that the saturation of the Cramer-Rao bound leads to the power spectrum at the scale of horizon exit in agreement with our former discussion on the duration of inflation. More precisely, it saturates Cramer-Rao for $\Delta \phi=\frac{H}{\sqrt{\epsilon}}$ that corresponds to $\Delta t=\frac{1}{\epsilon}$. In essence, the modes freeze when they saturate the minimal value for the variance. In order to estimate the tilt

$$
\frac{d \ln \left(\Delta^{2}\right)}{d \ln (k)}
$$

for $k$ the value at the horizon exit we use that the horizon exit takes place after a time order $\frac{1}{\epsilon}$ in Hubble units. Using now that $\Delta^{2} \sim \frac{1}{\epsilon}$ we get in this approximation

$$
\frac{d \ln \left(\Delta^{2}\right)}{d \ln (k)}=n_{s}-1 \sim-\epsilon
$$

in qualitative agreement with the expected result $[1,5]$.

Next we summarize the logic flow of our approach. Using quantum estimation theory we have defined a clock operator playing the role of a maximally efficient quantum estimator of time. Let us for simplicity denote $\delta T(t)$ the quantum fluctuations for this operator. The cosmological Fisher function, that we model using a quasi-de Sitter evolution during inflation, defines the variance of this clock operator i.e., $\langle\delta T \delta T\rangle$. This variance is what we identify with the power spectrum of curvature fluctuations at horizon exit.

It is important to point out that the former connection between the quantum variance of the quantum estimator and the corresponding power spectrum for curvature perturbations is independent of the statistical uncertainties that naturally appear in the standard Cramer-Rao formula for the variance of the estimator. The key reason is that the quantum Fisher function sets the quantum value of $\Delta^{2}$ for the quantum estimator univocally.

Finally, we can use the former Fisher function $F=\frac{\epsilon}{H^{2}}$ to define a Jeffreys prior probability on cosmological initial conditions

$$
J=\sqrt{F}=\sqrt{\epsilon} \sqrt{N_{0}} .
$$

From this point of view the prior probability of no inflation i.e., of $\epsilon=0$ is just zero and the highest probability appears when the number of e-foldings $\frac{1}{\epsilon}$ is of the order of the initial Shannon information. We leave for a future work a more careful analysis of this qualitative statistical argument and its relation to Bayesian statistics.

We thank Alan Heavens and Shao-Jiang Wang for useful comments. The work of C. G. was supported by Grants No. SEV-2016-0597, No. FPA2015-65480-P, and No. PGC2018-095976-B-C21. The work of R. J. is supported by Grant No. PGC2018-098866-B-I00. 
[1] N. Aghanim et al. (Planck Collaboration), arXiv:1807 .06209 .

[2] A. A. Starobinsky, Phys. Lett. 91B, 99 (1980); Adv. Ser. Astrophys. Cosmol. 3, 130 (1987).

[3] A. H. Guth, Phys. Rev. D 23, 347 (1981); Adv. Ser. Astrophys. Cosmol. 3, 139 (1987).

[4] A. D. Linde, Phys. Lett. 108B, 389 (1982); Adv. Ser. Astrophys. Cosmol. 3, 149 (1987).

[5] V. F. Mukhanov and G. V. Chibisov, Pis'ma Zh. Eksp. Teor. Fiz. 33, 549 (1981) [JETP Lett. 33, 532 (1981)].

[6] S. W. Hawking, Phys. Lett. 115B, 295 (1982).

[7] A. Albrecht and P. J. Steinhardt, Phys. Rev. Lett. 48, 1220 (1982); Adv. Ser. Astrophys. Cosmol. 3, 158 (1987).

[8] A. H. Guth and S. Y. Pi, Phys. Rev. Lett. 49, 1110 (1982).
[9] H. V. Peiris et al. (WMAP Collaboration), Astrophys. J. Suppl. 148, 213 (2003).

[10] M. G. A. Paris, Int. J. Quantum. Inform. 07, 125 (2009).

[11] C. Gomez, arXiv:1912.08549.

[12] C. Gomez, Phys. Rev. D 101, 065016 (2020).

[13] A. Bhattacharyya, S. Das, S. S. Haque, and B. Underwood, Phys. Rev. D 101, 106020 (2020).

[14] V. Vedral, Rev. Mod. Phys. 74, 197 (2002).

[15] G. W. Gibbons and S. W. Hawking, Phys. Rev. D 15, 2738 (1977).

[16] N. Arkani-Hamed, S. Dubovsky, A. Nicolis, E. Trincherini, and G. Villadoro, J. High Energy Phys. 05 (2007) 055.

[17] A. J. Stam, Some Inequalities Satisfied by the Quantities of Information of Fisher and Shannon: Information and Control (Elsevier, New York, 1959). 